\section{Molecular basis for GIGYF- Me31B complex assembly in 4EHP-mediated translational repression}

\author{
Daniel Peter, ${ }^{1,2,4}$ Vincenzo Ruscica, ${ }^{1,4}$ \\ Praveen Bawankar, ${ }^{1,3,4}$ Ramona Weber, ${ }^{1}$ \\ Sigrun Helms, ${ }^{1}$ Eugene Valkov, ${ }^{1}$ Cátia Igreja, ${ }^{1}$ \\ and Elisa Izaurralde ${ }^{1,5}$

\footnotetext{
${ }^{1}$ Department of Biochemistry, Max Planck Institute for Developmental Biology, D-72076 Tübingen, Germany;

${ }^{2}$ European Molecular Biology Laboratory, 38042 Grenoble Cedex

9, France; ${ }^{3}$ Institute of Molecular Biology, 55128 Mainz, Germany
}

GIGYF (Grb10-interacting GYF [glycine-tyrosine-phenylalanine domain]) proteins coordinate with 4EHP (eIF4E [eukaryotic initiation factor 4E] homologous protein), the DEAD (Asp-Glu-Ala-Asp)-box helicase Me31B/DDX6, and mRNA-binding proteins to elicit transcript-specific repression. However, the underlying molecular mechanism remains unclear. Here, we report that GIGYF contains a motif necessary and sufficient for direct interaction with Me31B/DDX6. A $2.4 \AA$ crystal structure of the GIGYF-Me31B complex reveals that this motif arranges into a coil connected to a $\beta$ hairpin on binding to conserved hydrophobic patches on the Me31B RecA2 domain. Structure-guided mutants indicate that 4EHP-GIGYF-DDX6 complex assembly is required for tristetraprolin-mediated down-regulation of an AU-rich mRNA, thus revealing the molecular principles of translational repression.

Supplemental material is available for this article.

Received May 28, 2019; revised version accepted July 18, 2019.

Initiation of translation by the eukaryotic initiation factor $4 \mathrm{E}$ (eIF4E) is regulated by competitor cap-binding proteins of the eIF4E family, such as the eIF4E homologous protein (4EHP; also known as eIF4E2) (Kong and Lasko 2012). 4EHP is responsible for the assembly of translational repressor complexes that inhibit mRNA expression in different biological contexts (Cho et al. 2005, 2006; Villaescusa et al. 2009; Chapat et al. 2017). 4EHP specifically associates with Grb10-interacting GYF (glycine-tyrosine-phenylalanine domain) protein 1 (GIGYF1) and

[Keywords: translational repression; DEAD-box helicases,

RNA regulation]

${ }^{4}$ These authors contributed equally to this work.

${ }^{5}$ Deceased April 30, 2018.

Corresponding authors: catia.igreja@tuebingen.mpg.de, eugene.valkov @tuebingen.mpg.de

Article published online ahead of print. Article and publication date are online at http://www.genesdev.org/cgi/doi/10.1101/gad.329219.119. Freely available online through the Genes \& Development Open Access option.
GIGYF2. These proteins possess an N-terminal 4EHPbinding region (4EHP-BR) and a central compacted GYF domain (Supplemental Fig. S1A; Ash et al. 2010; Peter et al. 2017) that mediates the interaction with ZNF598, tristetraprolin (TTP), or the microRNA (miRNA)-induced silencing complex-associated TNRC6 proteins (Morita et al. 2012; Fu et al. 2016; Schopp et al. 2017). These RNA-associated proteins recruit the 4EHP-GIGYF2 complex to specific mRNAs important for mouse embryonic development, cytokine mRNA expression, or repression of miRNA targets, respectively (Morita et al. 2012; Fu et al. 2016; Tollenaere et al. 2019).

GIGYF proteins do not simply bridge 4EHP to the RNA-associated proteins but rather participate directly in the repression mechanism (Peter et al. 2017). Human GIGYF2 regulates the expression of a subset of mRNAs via the recruitment of the CCR4-NOT complex (Amaya Ramirez et al. 2018). GIGYF proteins also associate with DDX6 (Me31B in Drosophila melanogaster $[\mathrm{Dm}]$ and Dhhlp in yeast) (Amaya Ramirez et al. 2018; Ruscica et al. 2019), an important regulator of gene expression (Ostareck et al. 2014; Wang et al. 2015; Lumb et al. 2017) that acts as translational repressor and enhancer of mRNA decapping (Coller et al. 2001; Radhakrishnan et al. 2016).

DDX6 orthologs are RNA-dependent ATPases of the DEAD (Asp-Glu-Ala-Asp)-box family that feature two globular RecA-like domains (RecA1 and RecA2) connected by a flexible linker. DEAD-box proteins use ATP binding and hydrolysis coupled to RNA binding to promote conformational transitions and remodeling of RNA and/ or ribonucleoprotein particles (mRNPs) (Ozgur et al. 2015b). DDX6 has restricted conformational flexibility and limited ATPase activity and requires stimulation by interacting factors (Mathys et al. 2014).

DDX6 assembles in mutually exclusive complexes with P-body components such as EDC3, LSM14A, PatL1, and the eIF4E transporter protein (4E-T) (Jonas and Izaurralde 2013). These proteins use different short linear motifs to associate with two binding pockets in the RecA2 domain of DDX6, referred to here as Phe-Asp-Phe (FDF) and Trp (W) pockets (Tritschler et al. 2008; Sharif et al. 2013; Ozgur et al. 2015a; Brandmann et al. 2018).

To elucidate how GIGYF proteins function together with DDX6 in the regulation of mRNA expression, we determined the crystal structure of an N-terminal conserved motif from $D m$ GIGYF that mediates direct binding to Me31B (Fig. 1A; Supplemental Fig. S1A). This binding motif, characterized by a Pro-Glu-Trp (PEW) sequence and a "split" FDF sequence, binds to Me31B in a unique manner. We further show that recruitment of DDX6 via GIGYF2 is required in human cells for efficient translational repression of an AU-rich reporter mRNA by TTP. Collectively, these data have advanced our understanding of the molecular principles governing the assembly of mRNPs that rely on the 4EHP-GIGYF complex and DDX6 proteins to posttranscriptionally regulate gene expression.

(C) 2019 Peter et al. This article, published in Genes \& Development, is available under a Creative Commons License (Attribution-NonCommercial 4.0 International), as described at http://creativecommons.org/licenses/by-nc/4.0/. 


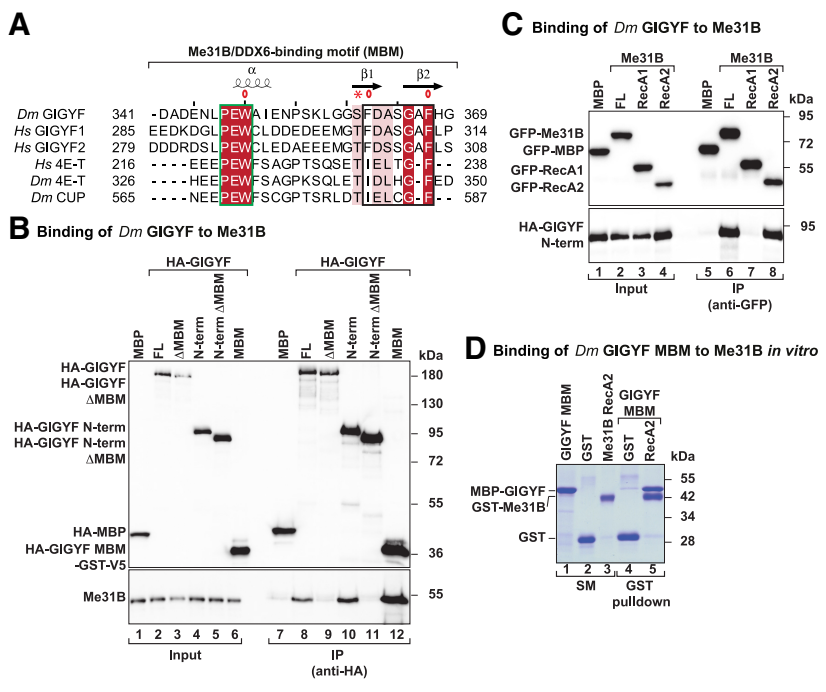

Figure 1. GIGYF proteins contain a conserved Me31B/DDX6-binding motif (MBM). (A) Sequence alignment of the MBM of $\mathrm{Dm}$ and Homo sapiens (Hs) GIGYF with the CUP homology domain (CHD) of $\mathrm{Dm}$ and $\mathrm{Hs}$ 4E-T and $\mathrm{Dm}$ CUP. Residues with $>70 \%$ similarity are shown with a light-colored background. Conserved residues are highlighted with a darker background and are printed in white. Secondary structure elements based on the structures presented in this study are indicated above the Dm GIGYF sequence. Boxed residues highlight the PEW (green) and FDF/IEL (black) motifs. The asterisk indicates the polar residue preceding the FDF motif. $(B)$ The binding of HA-Dm GIGYF (FL or the indicated proteins) to Me31B was analyzed in coimmunoprecipitation (co-IP) assays using anti-HA antibodies upon S2 cell transfection. HA-MBP served as a negative control. The input ( $1.5 \%$ for the HA proteins and $0.2 \%$ for Me $31 \mathrm{~B})$ and immunoprecipitated (30\% for the HA proteins and $45 \%$ for Me31B) fractions were analyzed by Western blotting using anti-HA and antiMe31B antibodies. (C) The interaction between GFP-Dm Me31B (FL or the indicated RecA domains) and HA-Dm GIGYF N-terminal expressed in S2 cells was analyzed in co-IP assays using anti-GFP antibodies. GFP-MBP served as a negative control. Input $13 \%$ for the GFP proteins and $1 \%$ for the HA proteins) and immunoprecipitated (15\% for the GFP proteins and $30 \%$ for the HA proteins) fractions were analyzed by Western blotting using anti-GFP and anti-HA antibodies. (D) GST pull-down assay showing the interaction between the GST-Me31B RecA2 domain and the MBP-Dm GIGYF MBM. GST served as a negative control. The starting material $16.25 \%$ for GST proteins and $2 \%$ for the MBP proteins) and bound fractions $(20 \%)$ were analyzed by SDS-PAGE followed by Coomassie blue staining. The size markers (in kilodaltons) are shown at the right of each panel.

\section{Results and Discussion}

The GIGYF linear motif is necessary and sufficient to directly bind Me31B/DDX6

The GIGYF orthologs contain a short conserved sequence motif with partial similarity to the CUP homology domain (CHD) present in 4E-T proteins (Fig. 1A; Kamenska et al. 2014; Ruscica et al. 2019). Deletion of this Me31B/DDX6-binding motif (MBM) abrogated the interaction of Me31B/DDX6 with transiently expressed and tagged GIGYF (Dm GIGYF and Homo sapiens $[\mathrm{Hs}]$ GIGYF1/2) in Drosophila and human cells (Fig. 1B; Supplemental Fig. S1B,C; Ruscica et al. 2019). The MBM alone interacted with Me31B/DDX6 as efficiently as full-length (FL) GIGYF or the N-terminal fragment of GIGYF (Fig. 1B; Supplemental Fig. S1B,C), indicating that the MBM is necessary and sufficient for a stable interaction between the proteins.
In coimmunoprecipitation (co-IP) assays, GIGYF proteins associated with the RecA2, but not the RecA1, domain of Dm Me31B and human DDX6 (Fig. 1C; Supplemental Fig. S1D,E), as observed previously for other DDX6-interacting factors (Tritschler et al. 2009; Sharif et al. 2013; Ozgur et al. 2015a; Brandmann et al. 2018). The purified recombinant GST-tagged RecA2 domain of Me31B/DDX6 associated with MBP-tagged Dm GIGYF and human GIGYF1/2 MBM by pull-down (Fig. 1D; Supplemental Fig. S1F,G). The MBM thus has a crucial role in mediating a direct and stable interaction between GIGYF and DDX6.

\section{The Dm GIGYF MBM interacts with Me31B using $a$ bipartite mode}

We hypothesized that the GIGYF MBM binds to the W pocket of DDX6 via the conserved PEW motif because of the apparent sequence similarity to the $\mathrm{CHD}$ region of 4E-T (Fig. 1A; Ozgur et al. 2015a). However, the presence of alanine or serine in place of the second phenylalanine in the FDF-like motif (FDA/S) and the absence of an Ile-GluLeu (IEL) motif as observed in 4E-T suggest that the binding mode to the conserved hydrophobic FDF pocket of DDX6 may have diverged. To investigate this further, we determined the crystal structure of the Dm GIGYF MBM (residues D343-G369) in complex with the RecA2 domain of Me31B (residues E264-V431) to $2.4 \AA$ resolution (Supplemental Table S1).

The RecA2 domain of Me31B adopts a typical $\alpha / \beta$-fold characterized by a central six-stranded parallel $\beta$ sheet covered by $\alpha$-helical layers on either side (Fig. 2A; Cheng et al. 2005). In the structure of the complex, the GIGYF MBM curves around helices $\alpha 10$ and $\alpha 11$ of Me31B to engage the conserved FDF and $\mathrm{W}$ pockets-known binding sites for Hs 4E-T, Hs and Saccharomyces cerevisiae (Sc) Edc3, Sc Pat1, and Hs LSM14A (Fig. 2A; Supplemental Fig. S2A-F; Tritschler et al. 2008; Sharif et al. 2013; Ozgur et al. 2015a; Brandmann et al. 2018). Two distinct structured elements can be identified in the MBM: a short coil running along helix $\alpha 11$ of Me31B and a $\beta$ hairpin containing a "split" FDF motif (FDx $\left.{ }_{4} \mathrm{~F}\right)$ (Fig. 2B-D).

The N-terminal PEW (P347, E348, and W349) peptide trio of the GIGYF MBM initiates a short coil that inserts the aromatic side chain of W349 ${ }^{\text {GIGYF }}$ (equivalent to W221 in 4E-T) into the hydrophobic pocket formed by residues V283, L310, L311, and F370 between helices a10 and a11 of Me31B (Fig. 2B; Supplemental Fig. S3A). Other DDX6 interactors also feature a large aromatic residue (W91 in Sc Edc3, F192 in Hs EDC3, F42 in Sc Pat1, or F396 in Hs LSM14A) inserted at the equivalent pocket of Dhh1/DDX6 (Supplemental Figs. S3, S4A-C). Hydrogen bonding between the side chains of Q306 ${ }^{\mathrm{Me} 31 \mathrm{~B}}$ and $\mathrm{K} 314^{\mathrm{Me} 31 \mathrm{~B}}$ and the backbone oxygens of N345 GIGYF and A $350^{\text {GIGYF }}$ lends additional stability to the interface (Fig. 2B).

The PEW sequence of the GIGYF MBM is then connected via a flexible linker to a $\beta$-hairpin structure formed at the FDF pocket of Me31B (Fig. 2A,C,D). The $\beta$ hairpin serves to orient the FDF motif (F361, D362, and F367 ${ }^{\text {GIGYF }}$ ) to optimally engage Me31B. The F361 and F367 $7^{\text {GIGYF }}$ are in positions structurally equivalent to those observed previously in other FDF or IEL sequences (Supplemental Fig. S5; Tritschler et al. 2008; Sharif et al. 2013; Ozgur et al. 2015a; Brandmann et al. 2018). The 


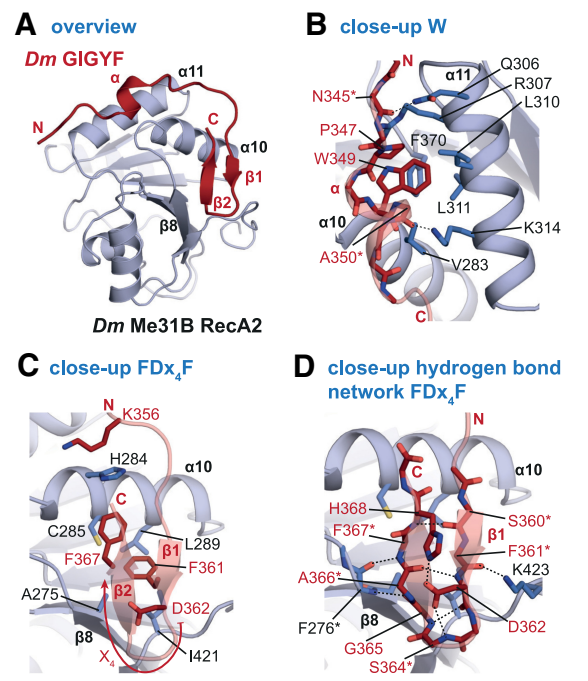

Figure 2. Structure of Dm GIGYF MBM bound to Me31B. (A) Overview of the structure of the Dm GIGYF MBM in complex with the Me31B RecA2 domain. Me31B is colored in light blue, and GIGYF is in red. Selected secondary structure elements are indicated. $(B)$ Close-up view on the PEW sequence of GIGYF at the W pocket of Me31B. $(C, D)$ Close-up views on the interactions of the $\mathrm{FDx}_{4} \mathrm{~F} \beta$ hairpin of GIGYF with Me31B. Selected interface residues are shown as sticks. For clarity reasons, all residues labeled with an asterisk are shown without their side chain.

aromatic rings of F361 and F367 GIGYF are accommodated in an "edge to face" orientation stabilized by a network of hydrophobic contacts formed by residues A275, H284, C285, L289, and I421 ${ }^{\text {Me31B }}$ (Fig. 2C). The side chain of D362 GIGYF forms hydrogen bonds to the backbone nitrogen of S364 ${ }^{\text {GIGYF }}$ as well as to the imidazole ring of $\mathrm{H} 368^{\mathrm{GIGYF}}$, linking the loop region to the C-terminal portion of the hairpin (Fig. 2D). G365 and F367 GIGYF participate in backbone-mediated interactions with $\mathrm{F} 276^{\mathrm{Me} 31 \mathrm{~B}}$, thus extending the Me $31 \mathrm{~B} \beta$ sheet at the tip of the $\beta 8$ strand (Fig. 2D). The first hairpin strand $(\beta 1)$ is anchored to Me31B by hydrogen bonds between the side chain of $\mathrm{K} 423^{\mathrm{Me} 31 \mathrm{~B}}$ and the backbone oxygen of F361 GIGYF (Fig. 2D).

The GIGYF MBM contacts two conserved surfaces on Dm Me31B in a composite bipartite binding arrangement that combines the salient features of the 4E-T PEW motif with the FDF motif present in EDC3 and LSM14A homologs as well as Sc Pat1 (Fig. 1A; Supplemental Figs. S3, S5). Thus, despite the overall conservation of the interface and the mutually exclusive binding, GIGYF exhibits notable structural differences compared with other DDX6 interactors by using a "split" FDx 4 F motif.

\section{The GIGYF FDx $\mathrm{F}_{4}$ motif does not block NOT1 binding to DDX6}

NOT1, the scaffold protein of the CCR4-NOT deadenylase complex, interacts with a surface of RecA2 domain adjacent to but not overlapping with the surface engaged by the other DDX6 interactors (Supplemental Fig. S2A; Chen et al. 2014; Mathys et al. 2014). Comparative structural analysis of DDX6-containing complexes indicates that the negatively charged residues preceding the FDF and DW motifs present in Hs EDC3 and PatL1 proteins (D203, F204, D205, and F206 ${ }^{\mathrm{EDC} 3}$ and D43, D44, D45, and $\mathrm{W} 46^{\mathrm{PatL} 1}$ ) (Supplemental S4A,B) are very likely to induce electrostatic repulsions to the NOT1 residues that face the DDX6 FDF pocket. This will impose a significant unfavorable energetic cost on the assembly of a ternary complex by EDC3/PatL1, DDX6, and NOT1 /Ozgur et al. 2015a). However, in the case of 4E-T, the IEL motif is preceded by a polar residue (Fig. 1A), which permits binding to the DDX6-CNOT1 complex. By analogy, GIGYF can, in principle, assemble into a ternary complex with DDX6-NOT1, as the residues located N-terminally to the $\mathrm{FDx}_{4} \mathrm{~F}$ motif are polar rather than negatively charged (Fig. 1A). We have not validated this structural hypothesis in cells, but an $\mathrm{N}$-terminal region of human GIGYF2 containing the MBM does bind NOT1 in co-IP assays in HeLa cells (Amaya Ramirez et al. 2018), thus providing support to the notion of the existence of a functional CNOT1-DDX6-GIGYF2 ternary complex.

The bipartite binding mode is essential for GIGYF-DDX6 complex assembly

Guided by the structural analysis of the binding interfaces, we next substituted key residues on the $\mathrm{W}$ (LK-AA mutant) or FDF (CL-AA mutant) pockets in Me31B/DDX6 (Supplemental Table S2; Supplemental Fig. S4D) and tested binding by co-IP following transient expression in either Dm S2 or human cells. The interaction of GIGYF (HA-Dm GIGYF or Hs GIGYF1/2) with Me31B/DDX6 was strongly impaired by individual or combined pocket mutations (Fig. 3A,B), pointing to a crucial functional role for both binding pockets in stabilizing the association between the proteins.

Conversely, we also analyzed the effect of amino acid substitutions in GIGYF on the interaction with DDX6. Tryptophan substitution by alanine in the PEW motif $\left(\mathrm{W}^{*}\right.$ mutant), of both phenylalanines in the $\mathrm{FDx}_{4} \mathrm{~F}$ motif (FF* mutant), or in combination (WFF* mutant) (Supplemental Table S2) abolished the interaction of $D m$ and human GIGYF with Me31B/DDX6 in cells (Fig. 3C,D; Supplemental Fig. S6A).

Dm HPat and human PatL1 do not contain an FDF motif but rather contain a DW sequence motif that interacts with Me31B/DDX6 (Supplemental Fig. S4A). Interestingly, the mutations in the $\mathrm{W}$ and FDF pockets of Me31B/ DDX6 also strongly reduced binding to HPat/PatL1, which is consistent with previous observations (Fig. 3A, B; Sharif et al. 2013). However, the disruption of the FDF pocket (CL-AA mutant) did not affect the association of Me31B/DDX6 with 4E-T or LSM14A and only mildly impaired binding to EDC3 (Dm and $\mathrm{Hs}$ ) (Fig. 3A; Supplemental Fig. S6B-D). In contrast, the substitutions in the $\mathrm{W}$ pocket strongly reduced binding to $\mathrm{Dm}$ and human 4E-T, EDC3, and LSM14A (Fig. 3A; Supplemental Fig. S6B-D).

Collectively, these binding studies are consistent with a differential contribution of the two binding pockets in DDX6 toward promoting stable interactions with various factors. Reported differences in the binding affinities further support this model: Both $S c$ Pat1 and Sc EDC3 are high-affinity binders of $S c$ Dhh1 $\left(K_{\mathrm{d}}\right.$ of $50 \mathrm{nM}$ and 200 $\mathrm{nM}$, respectively) (Sharif et al. 2013); human DDX6 interactors are rather more diverse in their affinities, with reported $K_{\mathrm{d}} \mathrm{s}$ of $230 \mathrm{nM}$ for PatL1, $390 \mathrm{nM}$ for 4E-T, 410 $\mathrm{nM}$ for EDC3, and 1.62 $\mu \mathrm{M}$ for LSM14A (Brandmann et al. 2018). 


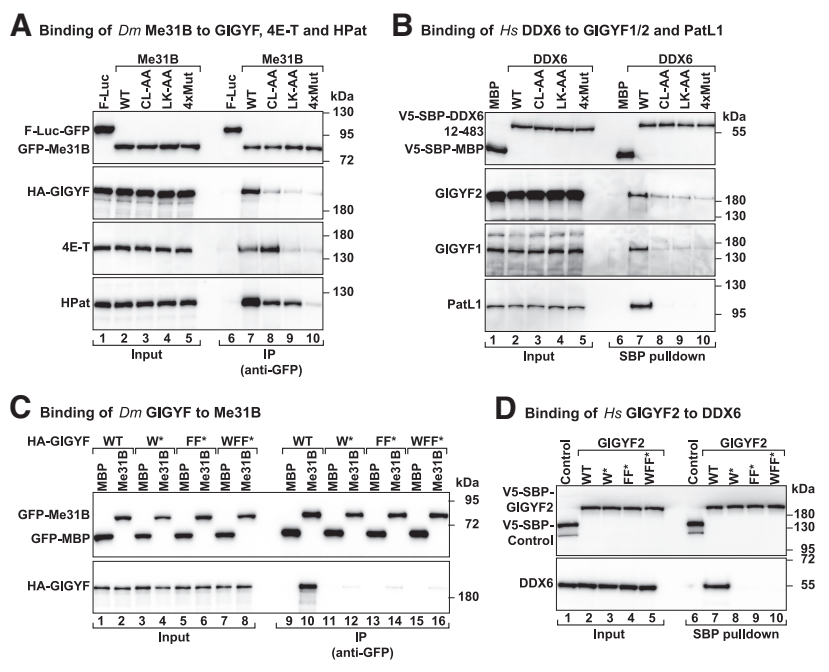

Figure 3. GIGYF proteins use a bipartite binding mode to address DDX6. $(A, B)$ Analysis of the interaction of GFP-Me31B with HAGIGYF, 4E-T, and HPat in S2 cells $(A)$ or of V5-SBP-DDX6 with human GIGYF1/2 and PatL1 $(B)$. The DDX6 proteins are either wild type (WT) or the indicated mutants. GFP proteins were immunoprecipitated using anti-GFP antibodies, whereas SBP proteins were pulled down using streptavidin-coated beads. Firefly luciferase (FLuc)-GFP and V5-SBP-MBP served as negative controls. (A) The inputs for the $D m$ experiment were $3 \%$ for the GFP proteins and $1 \%$ for HA-GIGYF, 4E-T, and HPat, whereas bound fractions corresponded to $15 \%$ for the GFP proteins, $30 \%$ for HA-GIGYF and $4 \mathrm{E}-\mathrm{T}$, and $20 \%$ for HPat. $(B)$ In the pull-down assay with the human proteins, inputs were $1.25 \%$ for the V5-SBP proteins and $0.5 \%$ for GIGYF1 $/ 2$ and PatL1, while bound fractions corresponded to $5 \%$ for the V5-SBP proteins and $30 \%$ for the other proteins. Samples were analyzed by Western blotting using anti-GFP, anti-HA, and anti-V5 antibodies and protein-specific antibodies. $(C)$ The interaction between GFPMe31B and HA-GIGYF-wild type or the indicated mutants $\left(\mathrm{W}^{*}\right.$ [W349A], FF* [F361A, F367A], and WFF*) (Supplemental Table S2)was analyzed in Dm S2 cell lysates using anti-GFP co-IP. GFP-MBP served as a negative control. The input $(3 \%$ for the GFP proteins and $1 \%$ for the HA proteins) and bound fractions (15\% for the GFP proteins and $30 \%$ for the HA proteins) were analyzed by Western blotting using the indicated antibodies. $(D)$ Streptavidin-based pull-down assays showing the association of SBP-V5-Hs GIGYF2-wild type or the indicated mutants (W* [W288A], FF* [F300A, F306A], and WFF*) (Supplemental Table S2)—and DDX6. V5-SBP-MBP-F-Luc-EGFP served as a negative control. The input $1.25 \%$ for the V5-SBP proteins and $0.5 \%$ for DDX6) and bound fractions (8\% for the V5-SBP proteins and $30 \%$ for DDX6) were analyzed by Western blotting using the indicated antibodies.

\section{GIGYF2-DDX6 interaction contributes to TTP-mediated translational repression}

To explore the functional relevance of the GIGYF-DDX6 interaction, we investigated the regulation of mRNA expression by TTP in human cells. TTP represses the expression of AU-rich transcripts via the recruitment of the 4EHP-GIGYF2 complex (Fu et al. 2016; Peter et al. 2017). To test the TTP-mediated repression in a reporter assay, we chose a Renilla luciferase (R-Luc) mRNA with two copies of the TNF- $\alpha$ mRNA AU-rich element (ARE) in the $3^{\prime}$ untranslated region (UTR) (Supplemental Fig. S7A). To distinguish the consequences of translational repression from degradation, an internal polyadenosine sequence of 90 nucleotides was "tailed" by a noncoding RNA MALAT1 sequence at the 3 ' end, which is generated by RNase P endonucleolytic cleavage, rendering this reporter mRNA resistant to $5^{\prime}-3^{\prime}$ decay (R-Luc-ARE-
A $_{90}$-MALAT1) (Peter et al. 2017). A plasmid encoding firefly luciferase (F-Luc-GFP) was included as a transfection and normalization control.

To bypass the recruitment of DDX6 via NOT1, we transiently expressed a TTP construct lacking the NOT1binding motif ( $\triangle \mathrm{CIM}$ ) (Fabian et al. 2013). We observed that TTP $\triangle$ CIM efficiently repressed the expression of the R-Luc reporter without altering its mRNA abundance in control cells (Fig. 4A; Supplemental Fig. S7B,C). By comparison, TTP-induced translational repression was alleviated in GIGYF1/2-null cells (GIGYF1/2 knockout) even though the observed level of TTP expression was comparable with that in the control cells (Fig. 4, A,B, lane 4 vs. lane 2). In GIGYF1/2-null cells, TTP-mediated translational repression was restored by coexpression of GIGYF2 and its stabilizing partner, 4EHP (Fig. 4A,B). However, the repressive function of TTP could be selectively impaired when 4EHP was coexpressed with the GIGYF2 mutants that do not bind to DDX6 (WFF*) or TTP (GYF*) (Fig. 4A,B; Supplemental Fig. S7D). The repressive function of TTP was critically dependent on the ARE, as none of the factors influenced the expression of a reporter lacking this sequence (R-Luc- $\mathrm{A}_{95}$-MALAT1) (Supplemental Fig. S7E,F). Collectively, these data support a model in which the assembly of the 4EHPGIGYF2-DDX6 complex is a prerequisite for TTP-mediated translational control of AU-rich transcripts.

\section{Concluding remarks}

In this study, we showed that GIGYF proteins interact directly with the RNA-dependent ATPase DDX6 via a short motif. This interaction is mutually exclusive with other DDX6-binding partners such as 4E-T, EDC3, LSM14A, and PatL1 and has an important functional role in posttranscriptional regulation (Fig. 4C). We showed that GIGYF2 is a direct link between DDX6 and TTP, which explains at the molecular level why DDX6 is required for ARE mRNA translational repression (Qi et al. 2012). The GIGYF-4EHP complex can also be part of TTP-independent mRNPs via direct mRNA binding (Amaya Ramirez et al. 2018) or the interaction with ZNF598 and TNRC6 proteins (Morita et al. 2012; Schopp et al. 2017). As the latter are important in ribosome quality control (Garzia et al. 2017; Sundaramoorthy et al. 2017; Juszkiewicz et al. 2018) and miRNA-mediated gene silencing (Chapat et al. 2017), respectively, the control of mRNA expression by the 4EHP-GIGYF-DDX6 complex is relevant for a wide range of cellular transcripts. Furthermore, as all of the components of the complex have been implicated to function in miRNA-mediated translational repression (Chen et al. 2014; Mathys et al. 2014; Chapat et al. 2017; Schopp et al. 2017), the 4EHPGIGYF-DDX6 complex is likely to have an important role in miRNA-mediated mechanisms.

\section{Materials and methods}

DNA constructs

The DNA constructs used in this study are described in the Supplemental Material and listed in Supplemental Table S2. All of the constructs and mutations were confirmed by sequencing.

\section{Protein production and purification}

The experimental procedures for the production and purification of recombinant proteins are described in the Supplemental Material. 


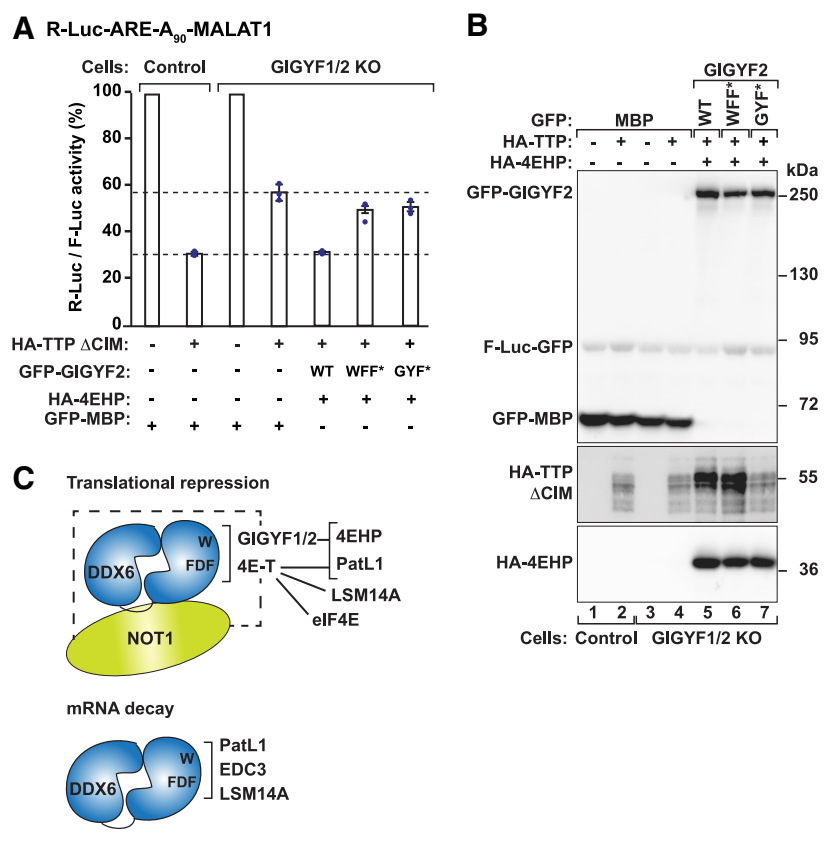

Figure 4. Recruitment of DDX6 by Hs GIGYF2 contributes to TTPmediated translational repression of an ARE mRNA reporter. $(A)$ Control or GIGYF1/2-null HEK293T cells (knockout [KO]) were transfected with the R-Luc-ARE-A $90-M A L A T 1$ reporter and plasmids expressing wild type (WT) or the indicated GIGYF2 mutants, HA4EHP, and HA-TTP $\triangle$ CIM (Fabian et al. 2013). An F-Luc-GFP reporter served as a transfection control. R-Luc activity was normalized to that of the F-Luc transfection control and set to $100 \%$ in the absence of TTP for each cell line. Bars represent the mean values, error bars represent standard deviations, and the blue dots represent the individual points from three independent experiments. $(B)$ Western blot analysis showing the expression of the proteins used in the complementation assay. Note that TTP $\triangle$ CIM is stabilized in GIGYF1/2 knockout cells expressing GIGYF wild type or WFF*. However, repression did not correlate with TTP levels but with the coexpression of a functional repressor complex. $(C)$ DDX6 complexes involved in translational repression and mRNA decay. GIGYF1/2, 4E-T, PatL1, EDC3, and LSM14A assemble mutually exclusive complexes with DDX6. NOT1 associates with a different surface of DDX6, but a ternary complex forms only in the presence of $4 \mathrm{E}-\mathrm{T}$ and possibly GIGYF1/2. Thus, DDX6 serves as a molecular adaptor for the assembly of protein complexes with distinct molecular roles-translational repression versus decapping. Independently of DDX6, 4E-T and GIGYF1/2 bind to NOT1, 4EHP, and PatL1. 4E-T is also an eIF4Eand an LSM14A-binding protein.

\section{Crystallization, data collection, and structure determination}

Detailed descriptions of the crystallization conditions and of the structure determination are in the Supplemental Material.

\section{Co-IP assays and Western blotting}

Co-IP assays in HEK293T and Schneider S2 cells were performed in the presence of RNase A as described previously (Peter et al. 2015a). All Western blots were developed using the ECL Western blotting detection system (GE Healthcare). The antibodies used in this study are listed in Supplemental Table S3.

\section{Pull-down assays}

The in vitro pull-down assays were performed as described previously (Igreja et al. 2014; Peter et al. 2015a,b). The details are in the Supplemental Material.

\section{Complementation assay}

HEK293T cells (wild-type or GIGYF1/2-null cells) were seeded in six-well plates $\left(0.6 \times 10^{6}\right.$ cells per well) and transfected using Lipofectamine 2000 (Invitrogen). The transfection mixtures contained $1 \mu \mathrm{g}$ of the R-Luc reporters and $0.25 \mu \mathrm{g}$ of the F-Luc control in the presence of $50 \mathrm{ng}$ of $\lambda \mathrm{N}$-HA-TTP $\Delta \mathrm{CIM}, 0.2 \mu \mathrm{g}$ of GFP-MBP, $1 \mu \mathrm{g}$ of GFP-GIGYF2 (wild type or mutants), or $0.5 \mu \mathrm{g}$ of $\lambda \mathrm{N}-\mathrm{HA}-4 \mathrm{EHP}$. F-Luc and R-Luc activities were measured $2 \mathrm{~d}$ after transfection using the dual-luciferase reporter assay system (Promega). RLuc activity was normalized to that of the F-Luc control and set to $100 \%$ in the absence of TTP in wild-type and GIGYF1/2-null cells. Total RNA was isolated using TriFast (Peqlab Biotechnologies), and the RNA samples were analyzed by Northern blot as described previously (Behm-Ansmant et al. 2006).

\section{Data availability}

Atomic coordinates and structure factors have been deposited in the Protein Data Bank under the accession codes 6S8R (Dm Me31B-GIGYF) and 6S8S (Hs DDX6-EDC3).

\section{Acknowledgments}

We dedicate this work to the memory of Elisa Izaurralde, who passed away while this manuscript was at the initial stage. We gratefully acknowledge that the study was conceived and carried out in her laboratory. We are thankful to C. Weiler, M.-Y. Chung, and G. Wagner for technical assistance; J. Braun for cloning the initial Hs DDX6 DNA constructs; and the staff at the PX beamline of the Swiss Light Source for support. We especially thank P. Lasko for kindly providing the anti-Dm 4E-T antibody. This work was supported by the Max Planck Society.

Author contributions: D.P. purified, crystallized, collected the data, and determined the structure of the complex. E.V. contributed to structural data analysis. R.W. performed the complementation assay. P.B., S.H., and V.R. performed co-IP or pull-down assays. P.B. generated several of the constructs used in this study, and V.R. contributed to complex purification. C.I. coordinated the project. E.I. was the principal investigator. D. P., E.V., and C.I. wrote the manuscript. All authors corrected the manuscript.

\section{References}

Amaya Ramirez CC, Hubbe P, Mandel N, Béthune J. 2018. 4EHP-independent repression of endogenous mRNAs by the RNA-binding protein GIGYF2. Nucleic Acids Res 46: 5792-5808. doi:10.1093/nar/ gky198

Ash MR, Faelber K, Kosslick D, Albert GI, Roske Y, Kofler M, Schuemann M, Krause E, Freund C. 2010. Conserved $\beta$-hairpin recognition by the GYF domains of Smy2 and GIGYF2 in mRNA surveillance and vesicular transport complexes. Structure 18: 944-954. doi:10.1016/j.str .2010.04.020

Behm-Ansmant I, Rehwinkel J, Doerks T, Stark A, Bork P, Izaurralde E. 2006. mRNA degradation by miRNAs and GW182 requires both CCR4:NOT deadenylase and DCP1:DCP2 decapping complexes. Genes Dev 20: 1885-1898. doi:10.1101/gad.1424106

Brandmann T, Fakim H, Padamsi Z, Youn J-Y, Gingras A-C, Fabian MR, Jinek M. 2018. Molecular architecture of LSM14 interactions involved in the assembly of mRNA silencing complexes. EMBO J 37: e97869. doi:10.15252/embj.201797869

Chapat C, Jafarnejad SM, Matta-Camacho E, Hesketh GG, Gelbart IA, Attig J, Gkogkas CG, Alain T, Stern-Ginossar N, Fabian MR, et al. 2017. Cap-binding protein 4EHP effects translation silencing by microRNAs. Proc Nat1 Acad Sci 114: 5425-5430. doi:10.1073/pnas .1701488114

Chen Y, Boland A, Kuzuoğlu-Öztürk D, Bawankar P, Loh B, Chang C-T, Weichenrieder O, Izaurralde E. 2014. A DDX6-CNOT1 complex and W-binding pockets in CNOT9 reveal direct links between miRNA target recognition and silencing. Mol Cell 54: 737-750. doi:10.1016/j .molcel.2014.03.034 
Cheng Z, Coller J, Parker R, Song H. 2005. Crystal structure and functional analysis of DEAD-box protein Dhh1p. RNA 11: 1258-1270. doi:10 $.1261 / \mathrm{rna} .2920905$

Cho PF, Poulin F, Cho-Park YA, Cho-Park IB, Chicoine JD, Lasko P, Sonenberg N. 2005. A new paradigm for translational control: inhibition via $5^{\prime}-3^{\prime}$ mRNA tethering by Bicoid and the eIF4E cognate 4EHP. Cell 121: 411-423. doi:10.1016/j.cell.2005.02.024

Cho PF, Gamberi C, Cho-Park YA, Cho-Park IB, Lasko P, Sonenberg N. 2006. Cap-dependent translational inhibition establishes two opposing morphogen gradients in Drosophila embryos. Curr Biol 16: 20352041. doi:10.1016/j.cub.2006.08.093

Coller JM, Tucker M, Sheth U, Valencia-Sanchez MA, Parker R. 2001. The DEAD box helicase, Dhh1p, functions in mRNA decapping and interacts with both the decapping and deadenylase complexes. RNA 7: 1717-1727. doi:10.1017/S135583820101994X

Fabian MR, Frank F, Rouya C, Siddiqui N, Lai WS, Karetnikov A, Blackshear PJ, Nagar B, Sonenberg N. 2013. Structural basis for the recruitment of the human CCR4-NOT deadenylase complex by tristetraprolin. Nat Struct Mol Biol 20: 735-739. doi:10.1038/nsmb .2572

Fu R, Olsen MT, Webb K, Bennett EJ, Lykke-Andersen J. 2016. Recruitment of the 4EHP-GYF2 cap-binding complex to tetraproline motifs of tristetraprolin promotes repression and degradation of mRNAs with AU-rich elements. RNA 22: 373-382. doi:10.1261/rna.054833 .115

Garzia A, Jafarnejad SM, Meyer C, Chapat C, Gogakos T, Morozov P, Amiri M, Shapiro M, Molina H, Tuschl T, et al. 2017. The E3 ubiquitin ligase and RNA-binding protein ZNF598 orchestrates ribosome quality control of premature polyadenylated mRNAs. Nat Commun 8: 16056. doi:10.1038/ncomms16056

Igreja C, Peter D, Weiler C, Izaurralde E. 2014. 4E-BPs require non-canonical 4E-binding motifs and a lateral surface of eIF4E to repress translation. Nat Commun 5: 4790. doi:10.1038/ncomms5790

Jonas S, Izaurralde E. 2013. The role of disordered protein regions in the assembly of decapping complexes and RNP granules. Genes Dev 27: 2628-2641. doi:10.1101/gad.227843.113

Juszkiewicz S, Chandrasekaran V, Lin Z, Kraatz S, Ramakrishnan V, Hegde RS. 2018. ZNF598 is a quality control sensor of collided ribosomes. Mol Cell 72: 469-481.e7. doi:10.1016/j.molcel.2018.08.037

Kamenska A, Lu W-T, Kubacka D, Broomhead H, Minshall N, Bushell M, Standart N. 2014. Human 4E-T represses translation of bound mRNAs and enhances microRNA-mediated silencing. Nucleic Acids Res 42: 3298-3313. doi:10.1093/nar/gkt1265

Kong J, Lasko P. 2012. Translational control in cellular and developmental processes. Nat Rev Genet 13: 383-394. doi:10.1038/nrg3184

Lumb JH, Li Q, Popov LM, Ding S, Keith MT, Merrill BD, Greenberg HB, Li JB, Carette JE. 2017. DDX6 represses aberrant activation of interferonstimulated genes. Cell Rep 20: 819-831. doi:10.1016/j.celrep.2017.06 .085

Mathys H, Basquin J, Ozgur S, Czarnocki-Cieciura M, Bonneau F, Aartse A, Dziembowski A, Nowotny M, Conti E, Filipowicz W. 2014. Structural and biochemical insights to the role of the CCR4-NOT complex and DDX6 ATPase in microRNA repression. Mol Cell 54: 751-765. doi:10.1016/j.molcel.2014.03.036

Morita M, Ler LW, Fabian MR, Siddiqui N, Mullin M, Henderson VC, Alain T, Fonseca BD, Karashchuk G, Bennett CF, et al. 2012. A novel 4EHP-GIGYF2 translational repressor complex is essential for mammalian development. Mol Cell Biol 32: 3585-3593. doi:10.1128/MCB .00455-12

Ostareck DH, Naarmann-de Vries IS, Ostareck-Lederer A. 2014. DDX6 and its orthologs as modulators of cellular and viral RNA expression. Wiley Interdiscip Rev RNA 5: 659-678. doi:10.1002/wrna.1237

Ozgur S, Basquin J, Kamenska A, Filipowicz W, Standart N, Conti E. 2015a. Structure of a human 4E-T/DDX6/CNOT1 complex reveals the differ- ent interplay of DDX6-binding proteins with the CCR4-NOT complex. Cell Rep 13: 703-711. doi:10.1016/j.celrep.2015.09.033

Ozgur S, Buchwald G, Falk S, Chakrabarti S, Prabu JR, Conti E. 2015b. The conformational plasticity of eukaryotic RNA-dependent ATPases. FEBS J 282: 850-863. doi:10.1111/febs.13198

Peter D, Igreja C, Weber R, Wohlbold L, Weiler C, Ebertsch L, Weichenrieder O, Izaurralde E. 2015a. Molecular architecture of 4E-BP translational inhibitors bound to eIF4E. Mol Cell 1074-1087. doi:10.1016/j .molcel.2015.01.017

Peter D, Weber R, Köne C, Chung MY, Ebertsch L, Truffault V, Weichenrieder O, Igreja C, Izaurralde E. 2015b. Mextli proteins use both canonical bipartite and novel tripartite binding modes to form eIF4E complexes that display differential sensitivity to 4E-BP regulation. Genes Dev 29: 1835-1849. doi:10.1101/gad.269068.115

Peter D, Weber R, Sandmeir F, Wohlbold L, Helms S, Bawankar P, Valkov E, Igreja C, Izaurralde E. 2017. GIGYF1/2 proteins use auxiliary sequences to selectively bind to 4EHP and repress target mRNA expression. Genes Dev 31: 1147-1161. doi:10.1101/gad.299420.117

Qi M-Y, Wang Z-Z, Zhang Z, Shao Q, Zeng A, Li X-Q, Li W-Q, Wang C, Tian F-J, Li Q, et al. 2012. AU-rich-element-dependent translation repression requires the cooperation of tristetraprolin and RCK/P54. Mol Cell Biol 32: 913-928. doi:10.1128/MCB.05340-11

Radhakrishnan A, Chen Y-H, Martin S, Alhusaini N, Green R, Coller J. 2016. The DEAD-box protein Dhhlp couples mRNA decay and translation by monitoring codon optimality. Cell 167: 122-132.e9. doi:10 .1016/j.cell.2016.08.053

Ruscica V, Bawankar P, Peter D, Helms S, Igreja C, Izaurralde E. 2019. Direct role for the Drosophila GIGYF protein in 4EHP-mediated mRNA repression. Nucleic Acids Res 47: 7035-7048. doi:10.1093/ nar/gkz429

Schopp IM, Amaya Ramirez CC, Debeljak J, Kreibich E, Skribbe M, Wild K, Béthune J. 2017. Split-BioID a conditional proteomics approach to monitor the composition of spatiotemporally defined protein complexes. Nat Commun 8: 15690. doi:10.1038/ncomms15690

Sharif H, Ozgur S, Sharma K, Basquin C, Urlaub H, Conti E. 2013. Structural analysis of the yeast Dhh1-Patl complex reveals how Dhh1 engages Pat1, Edc3 and RNA in mutually exclusive interactions. Nucleic Acids Res 41: 8377-8390. doi:10.1093/nar/gkt600

Sundaramoorthy E, Leonard M, Mak R, Liao J, Fulzele A, Bennett EJ. 2017. ZNF598 and RACK1 regulate mammalian ribosome-associated quality control function by mediating regulatory $40 \mathrm{~S}$ ribosomal ubiquitylation. Mol Cell 65: 751-760.e4. doi:10.1016/j.molcel.2016.12.026

Tollenaere MAX, Tiedje C, Rasmussen S, Nielsen JC, Vind AC, Blasius M, Batth TS, Mailand N, Olsen JV, Gaestel M, et al. 2019. GIGYF1/2-driven cooperation between ZNF598 and TTP in posttranscriptional regulation of inflammatory signaling. Cell Rep 26: 3511-3521.e4. doi:10 .1016/j.celrep.2019.03.006

Tritschler F, Eulalio A, Helms S, Schmidt S, Coles M, Weichenrieder O, Izaurralde E, Truffault V. 2008. Similar modes of interaction enable Trailer Hitch and EDC3 to associate with DCP1 and Me31B in distinct protein complexes. Mol Cell Biol 28: 6695-6708. doi:10.1128/MCB .00759-08

Tritschler F, Braun JE, Eulalio A, Truffault V, Izaurralde E, Weichenrieder O. 2009. Structural basis for the mutually exclusive anchoring of $P$ body components EDC3 and Tral to the DEAD box protein DDX6/ Me31B. Mol Cell 33: 661-668. doi:10.1016/j.molcel.2009.02.014

Villaescusa JC, Buratti C, Penkov D, Mathiasen L, Planagumà J, Ferretti E, Blasi F. 2009. Cytoplasmic Prepl interacts with 4EHP inhibiting Hoxb4 translation. PLoS One 4: e5213. doi:10.1371/journal.pone .0005213

Wang Y, Arribas-Layton M, Chen Y, Lykke-Andersen J, Sen GL. 2015. DDX6 orchestrates mammalian progenitor function through the mRNA degradation and translation pathways. Mol Cell 60: 118-130. doi:10.1016/j.molcel.2015.08.014 


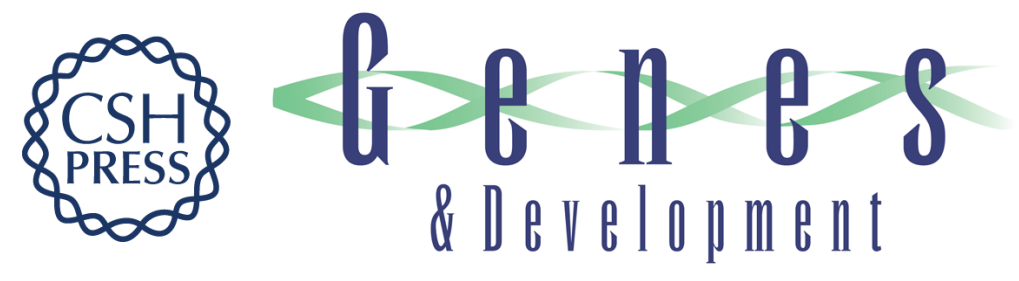

\section{Molecular basis for GIGYF-Me31B complex assembly in 4EHP-mediated translational repression}

Daniel Peter, Vincenzo Ruscica, Praveen Bawankar, et al.

Genes Dev. 2019, 33: originally published online August 22, 2019

Access the most recent version at doi:10.1101/gad.329219.119

\section{Supplemental http://genesdev.cshlp.org/content/suppl/2019/08/19/gad.329219.119.DC1 Material}

References This article cites 38 articles, 11 of which can be accessed free at: http://genesdev.cshlp.org/content/33/19-20/1355.full.html\#ref-list-1

Creative This article, published in Genes \& Development, is available under a Creative Commons Commons License (Attribution-NonCommercial 4.0 International), as described at License http://creativecommons.org/licenses/by-nc/4.0/.

Email Alerting Receive free email alerts when new articles cite this article - sign up in the box at the top Service right corner of the article or click here.

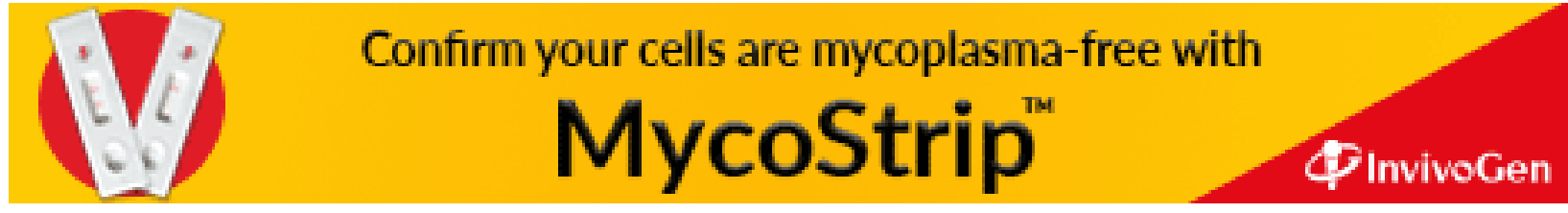

